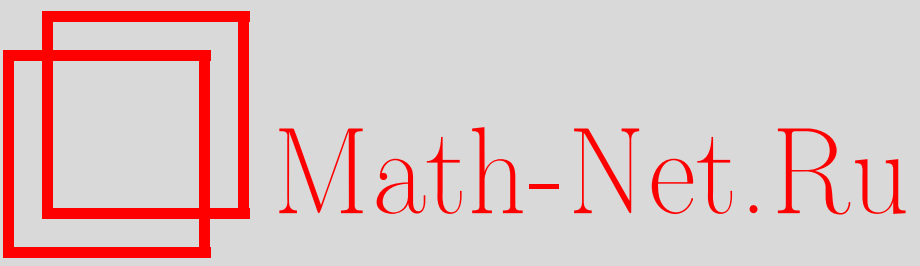

А. А. Туганбаев, Плоские мультипликационные модули, УМH, 2005, том 60, выпуск 1, 173-174

DOI: https://doi.org/10.4213/rm1399

Использование Общероссийского математического портала Math-Net.Ru подразумевает, что вы прочитали и согласны с пользовательским соглашением

http://www.mathnet.ru/rus/agreement

Параметры загрузки:

IP : 54.92.164.108

26 апреля 2023 г., 15:22:56 


\section{ПЛОСКИЕ МУЛЬТИПЛИКАЦИОННЫЕ МОДУЛИ}

\section{А. А. ТУГАНБАЕВ}

Все кольца предполагаются ассоциативными и с ненулевой единицей. Колцо назьвается инвариантным справа (слева), если все его правые (левые) идеалы являются идеалами. Выражения типа "инвариантное кольо" означают, что соответствующие условия выполнены справа и слева. Модуль $M_{A}$ называется плоским, если для любого левого $A$-модуля $L$ естественный групповой гомоморфизм $M \otimes_{A} L$ является мономорфизмом. Модуль $M_{A}$ называется мультипликационным, если для каждого его подмодуля $N$ существует такой идеал $B$ кольца $A$, что $N=M B$. Идеал $X$ кольца $A$ назьвается чистым слев $a$, если $x \in X x$ для каждого элемента $x \in X$. Если $X$ - подмножество модуля $M_{A}$, то $r(X)$ обозначает правый аннулятор множества $X$ в $A$. Если $M$ - мультипликационный модуль над коммутативным кольцом и идеал $r(M)$ чист, то $M$ плосок [1]. Основной резултат работы - теорема 1.

Теорема 1. Пусть А-инвариантное кольцо с коммутативным умножением идеалов и $M-$ мультипликационный правый $A$-модуль. Если идеал $r(M)$ чист слева, то М плосок.

Модуль $M_{A}$ назьвается модулем без $H$-кручения, если для любых $m \in M$ и $a \in A$ таких, что $m a=0$, существуют такие $x_{1}, \ldots, x_{n} \in M$ и $y_{1}, \ldots, y_{n} \in A$, что $m=\sum_{i=1}^{n} x_{i} y_{i}$ и $y_{i} a=0$ для всех $i$.

Лемма $2[2$; предложение 6.2 , теорема 6.3]. Правый $A$-модуль $M$ является плоским $\Longleftrightarrow$ для любого левого идеала $B$ кольца $A$ естественный групповой эпиморфизм $M \otimes_{A} B \rightarrow$

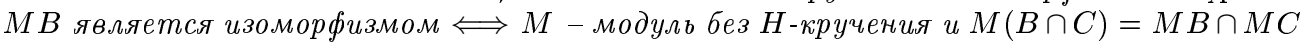
для любых левых идеалов $B$ и $C$ кольца $A$.

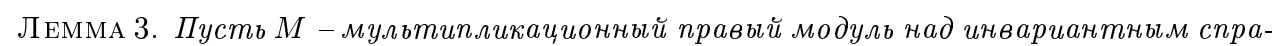
ва кольцом $A$ и идеал $r(M)$ чист слева.

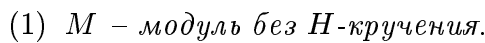

(2) Если $M(B \cap C)=M B \cap M C$ для любых двух левых идеалов $B$ и $C$ кольца $A$, то $M$ плосок.

ДокаЗАтельство. (1) Пусть $m \in M, a \in A$ и $m a=0$. Так как $A$ инвариантно справа, то $A a \subseteq a A \subseteq r(m)$. Поэтому $m A a=0$. Так как $M$ - мулштипликационньй модуль, то существует такой идеал $B$, что $M B=m A$. Тогда $M B a=m A a=0$. Поэтому $B a \subseteq r(M)$. Так как $M B=$ $m A$, то существуют такие $x_{1}, \ldots, x_{n} \in M$ и $b_{1}, \ldots, b_{n} \in B$, что $m=\sum_{i=1}^{n} x_{i} b_{i}$. Так как $b_{1} a, \ldots, b_{n} a \in r(M)$, то по условию существуют такие $c_{1}, \ldots, c_{n} \in r(M)$, что $c_{i} b_{i} a=b_{i} a$ $(i=1, \ldots, n)$. Положим $y_{i}=\left(1-c_{i}\right) b_{i}$. Так как $x_{i} c_{i} \in M r(M)=0(i=1, \ldots, n)$, то $m=$ $\sum_{i=1}^{n} x_{i}\left(1-c_{i}\right) b_{i}=\sum_{i=1}^{n} x_{i} y_{i}$ и $y_{i} a=\left(1-c_{i}\right) b_{i} a=b_{i} a-c_{i} b_{i} a=0(i=1, \ldots, n)$.

(2) Утверждение следует из (1) и леммы 2.

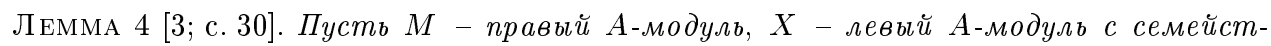
вом образующих $\left\{x_{i}\right\}_{i \in I}$ u $\left\{m_{i}\right\}_{i \in I}$ - такое подмножество модуля $M$, что почти все әлементы $m_{i}$ равны нулю. Тогда әлемент $\sum_{i \in I} m_{i} \otimes x_{i}$ группь $M \otimes_{A} X$ равен нулю в точности тогда, когда существуют конечное множество $\left\{y_{j}\right\}_{j \in J} \subseteq M$ и мноэкество $\left\{a_{j i}\right\}_{(j, i) \in J \times I} \subseteq A$ такие, что $a_{j i}=0$ для почти всех $(j, i), \sum_{i \in I} a_{j i} x_{i}=0$ для всех $j \in J$ и $m_{i}=\sum_{j \in J} y_{j} a_{j i}$ для всех $i \in I$.

Лемма 5. Пусть $M-$ правый $A$-модуль и идеал $r(M)$ чист слева.

(1) Для любого левого идеала $B$ кольца $A$ естественные групповые гомоморфизмы $M \otimes_{A} B \stackrel{f}{\longrightarrow} M \otimes_{A}(B+r(M)) \stackrel{g}{\longrightarrow} M \otimes_{A / r(M)}(B+r(M)) / r(M)$ являются изоморфизмами. Кроме того, если естественный групповой эпиморфизм

Работа выполнена при поддержке Российского фонда фундаменталшных исследований. 
$M \otimes_{A / r(M)}(B+r(M)) / r(M) \rightarrow M_{A / r(M)}(B+r(M)) / r(M)-$ изоморфизм, то естественный групповой эпиморфизм $M \otimes_{A} B \rightarrow M B-$ изоморфизм.

(2) Если $M_{A / r(M)}$ плосок, то $M_{A}$ плосок.

(3) Если $A$ инвариантно справа и $M(B \cap C)=M B \cap M C$ для любых левых идеалов В и $C$ кольца $A$, содержащих $r(M)$, то $M_{A}$ плосок.

ДокаЗАТеЛЬСтво. (1) Пусть $\sum_{i=1}^{n} m_{i} \otimes\left(b_{i}+c_{i}\right) \in M \otimes_{A}(B+r(M))$, где $m_{i} \in M$, $b_{i} \in B$ и $c_{i} \in r(M)$ для всех $i$. Так как идеал $r(M)$ чист слева, то существуют такие $d_{i} \in r(M)$, что $c_{i}=d_{i} c_{i}$ для всех $i$. Тогда $\sum_{i=1}^{n} m_{i} \otimes\left(b_{i}+c_{i}\right)=\sum_{i=1}^{n} m_{i} \otimes b_{i}+\sum_{i=1}^{n} m_{i} \otimes d_{i} c_{i}=$ $\sum_{i=1}^{n} m_{i} \otimes b_{i}+\sum_{i=1}^{n} m_{i} d_{i} \otimes c_{i}=\sum_{i=1}^{n} m_{i} \otimes b_{i}+\sum_{i=1}^{n} 0 \otimes c_{i}=\sum_{i=1}^{n} m_{i} \otimes b_{i}$. Поэтому $f-$ эпиморфизм. Докажем, что $f$ - изоморфизм. Пусть $\alpha$ - естественный мономорфизм из $B$ в $B+$ $r(M)$ и $\sum_{i=1}^{n} m_{i} \otimes b_{i} \in M \otimes_{A} B$, где $m_{i} \in M$ и $b_{i} \in B$ для всех $i$. Тогда $f\left(\sum_{i=1}^{n} m_{i} \otimes b_{i}\right)=$ $\sum_{i=1}^{n} m_{i} \otimes \alpha\left(b_{i}\right)$. Допустим, что $\sum_{i=1}^{n} m_{i} \otimes \alpha\left(b_{i}\right)=0$. Положим $x_{i}=\alpha\left(b_{i}\right)$ при $i \in\{1, \ldots, n\}$. Существует такое множество $I$, что $\{1, \ldots, n\} \subseteq I$ и $B+r(M)=\left\{x_{i}\right\}_{i \in I}$. Положим $m_{i}=0$ при $i \in I \backslash\{1, \ldots, n\}$. Тогда элемент $\sum_{i \in I} m_{i} \otimes x_{i}$ группы $M \otimes_{A}(B+r(M))$ равен нулю. По лемме 4 сушествуют конечное множество $\left\{y_{j}\right\}_{j \in J} \subseteq M$ и множество $\left\{a_{j i}\right\}_{(j, i) \in J \times I} \subseteq A$ такие, что $a_{j i}=0$ для почти всех $(j, i), \sum_{i \in I} a_{j i} x_{i}=0$ для всех $j \in J$ и $m_{i}=\sum_{j \in J} y_{j} a_{j i}$ для всех $i \in I$. Тогда $\sum_{i=1}^{n} m_{i} \otimes b_{i}=\sum_{i \in I} m_{i} \otimes b_{i}, \sum_{i \in I} a_{j i} b_{i}=0$ для всех $j \in J$ и элемент $\sum_{i=1}^{n} m_{i} \otimes b_{i}$ группы $M \otimes_{A} B$ равен $\sum_{i \in I}\left(\sum_{j \in J} y_{j} a_{j i}\right) \otimes b_{i}=\sum_{j \in J} y_{j} \otimes\left(\sum_{i \in I} a_{j i} b_{i}\right)=0$. Поэтому $f$ - изоморфизм. Непосредственно проверяется, что $g$ - изоморфизм. Таким образом, в естественной коммутативной диаграмме

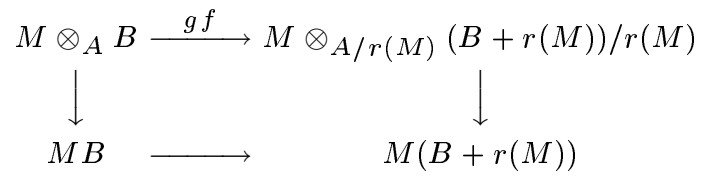

верхняя стрелка - изоморфизм. Кроме того, очевидно, что нижняя стрелка - изоморфизм. Поэтому если правая стрелка этой диаграммы - изоморфизм, то и левая стрелка $M \otimes_{A} B \rightarrow M B-$ изоморфииз.

(2) Утверждение следует из (1) и леммы 2.

(3) По лемме $3(2) M_{A / r(M)}$ плосок. По (2) $M_{A}$ плосок.

Лемма 6 [4]. Пусть $A-$ кольцо с коммутативным умножением правых идеалов и

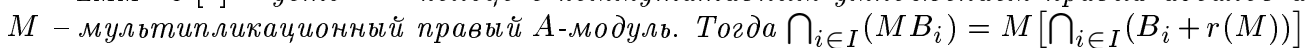
для каждого непустого набора правых идеалов $\left\{B_{i}\right\}_{i \in I}$ кольца $A$.

ОКОНЧАНИЕ ДОКАЗАТЕЛЬСТВА ТЕОРЕМЫ 1 . По лемме $6 \quad M(B \cap C)=M B \cap M C$ для любых левых идеалов $B$ и $C$ из $A$. По лемме $5(3) M$ плосок.

\section{СПИСОК ЛИТЕРАТУРЫ}

[1] A. G. Naoum // Period. Math. Hungar. 1990. V. 21. № 4. P. 309-317. [2] A. A. Tuganbaev. Semidistributive Modules and Rings. Dordrecht: Kluwer, 1998. [3] B. Stenström. Rings of Quotients: An Introduction to Methods of Ring Theory. New York: Springer-Verlag, 1975. [4] A. A. Tuganbaev // J. Math. Sci. 2004. V. 123. № 2. P. 3839-3905.

Московский энергетический институт (технический университет)

E-mail: askar@tuganbaev.mccme.ru
Представлено А. В. Михалевьм Принято редколлегией 23.11.2004 